Meta

Journal des traducteurs

Translators' Journal

\title{
UREF RLTT : Deuxièmes Journées scientifiques, Mons 25-27
} avril 1991

\section{L'environnement traductionnel : la station de travail du} traducteur de l'an 2001

\section{Philippe Thoiron}

Volume 37, numéro 2, juin 1992

URI : https://id.erudit.org/iderudit/004479ar

DOI : https://doi.org/10.7202/004479ar

Aller au sommaire du numéro

Éditeur(s)

Les Presses de l'Université de Montréal

ISSN

0026-0452 (imprimé)

1492-1421 (numérique)

Découvrir la revue

Citer ce document

Thoiron, P. (1992). UREF RLTT : Deuxièmes Journées scientifiques, Mons 25-27 avril 1991 : l'environnement traductionnel : la station de travail du traducteur de l'an 2001. Meta, 37(2), 384-386. https://doi.org/10.7202/004479ar d'utilisation que vous pouvez consulter en ligne. 


\section{UREF \\ RLTT : DEUXIËMES JOURNÉES \\ SCIENTIFIQUES, MONS, 25-27 AVRIL 1991 \\ L'ENVIRONNEMENT TRADUCTIONNEL: LA STATION DE TRAVAIL DU TRADUCTEUR DE L'AN 2001}

L'Université des réseaux d'expression française (UREF), créée par l'Assemblée générale de l'AUPELF (Association des universités partiellement ou entièrement de langue française), a pour ambition de mailler, de mettre en réseau la communauté scientifique et technique d'expression française. Des réseaux thématiques de recherche partagée rassemblent des chercheurs et des institutions (composantes d'universités ou d'écoles) sur un thème commun, en vue d'objectifs spécifiques. C'est l'un de ces réseaux thématiques, le Réseau lexicologie terminologie Traduction qui a tenu à Mons (Belgique) du 25 au 27 avril 1991 ses Deuxièmes Journées scientifiques.

Ce colloque international, qui s'est déroulé dans les locaux de l'École d'interprètes internationaux et a bénéficié du concours de l'Institut de linguistique de l'Université de Mons-Hainaut et de la collaboration de l'Agence de coopération culturelle et technique, avait pour thème «L'environnement traductionnel : la station de travail du traducteur de l'an 2001». Un public nombreux de traductcurs, terminologues, rédacteurs, interprètes, mais aussi de décideurs d'organismes, privés ou publics, du nord comme du sud, a pu écouter plus de trente communications d'orateurs venus d'une quinzaine de pays (Afrique, Amérique du Nord et Europe). Ont ainsi pu présenter leurs travaux les chercheurs universitaires et les concepteurs de logiciels traductionnels, mais aussi les spécialistes de l'enseignement des langues et des disciplines langagières.

L'explosion des connaissances, la volonté et la nécessité de communiquer sont telles dans la communauté techno-scientifique d'aujourd'hui que la traduction est l'objet de pressions considérables : ce n'est déjà plus la profession artisanale que certains participants se remémoraient avec nostalgie, mais ce n'est pas encore l'activité industrielle mécanisée et déshumanisée que d'autres redoutent. La masse des documents à traiter ne cesse de croître et tout laisse prévoir une augmentation exponentielle du fait des progrès techniques et scientifiques déjà évoqués, bien sûr, mais aussi des mutations politiques, engagées ou prévisibles. Si chacun a bien conscience qu'il faudra traduire plus, c'est la question des moyens et de la formation offerts aux traducteurs de l'an 2001 qui a été au centre des travaux du colloque.

Des moyens existent déjà dans le domaine de l'aide informatique au traducteur. Diverses solutions ont été présentées, allant de la consultation de dictionnaires électroniques en ligne jusqu'au recours à des systèmes plus 
ambitieux visant la traduction automatique de textes empruntés à des domaines très restreints, comme ceux de la météorologie par exemple. Dans leur ensemble, les participants ont pu mesurer la distance qui reste à parcourir. Ils ont pu constater, à travers les diverses communications, que les difficultés d'origine informatique, si souvent évoquées dans un passé encore récent (capacité mémoire, vitesse de calcul, etc.), n'étaient plus guère mentionnées mais que les problèmes linguistiques avaient pris le relais, avec une acuité peut-être décuplée par le trop long silence qui les a entourés.

Chacun a bien dû se convaincre qu'on ne pourra plus faire l'économie de réflexions théoriques approfondies, multiples et convergentes. Qu'il s'agisse de morphologie, de syntaxe, de lexique général ou de terminologie. 11 semble bien que les transferts entre langue source et langue cible devront faire appel à des descripteurs sémantico-cognitifs. De la même manière, la structuration des notions dans les domaines de haute spécialité doit permettre au traducteur de mieux maîtriser le fragment qui le concerne à un moment donné : la quête de l'information qui lui est indispensable n'est possible que si une réflexion théorique sur l'organisation des réseaux notionnels et leur relation avec les systèmes de désignation a bien été conduite.

Ces préoccupations de linguistique théorique, et leur lien avec les cognisciences, ont été perçues comme d'autant plus importantes qu'elles doivent commander à la relation entre le traducteur et sa station de travail. Que celle-ci existe déjà, ou qu'on l'imagine pour un avenir proche, elle s'organise toujours autour des mêmes objets, matériels et logiciels. On évoque un microordinateur puissant, rapide, doté d'un ou de plusieurs disques rigides, supportant des logiciels de traitement de textes, de gestion de bases de données contenant plusieurs dictionnaires spécialisés, de télécommunications, de traduction assistée et éventuellement de reconnaissance optique de caractères. Le .micro-ordinateur peut être intégré à un réseau et relié à un lecteur de disque optique et à une imprimante laser de qualité. La station de travail est complétée par un télécopieur.

L'abondance technologique fait évidemment naître la crainte, maintes fois exprimée au cours de ces journées, de voir le traducteur réduit au rôle de servant d'une machinerie qui le dépasserait ou qu'il ne maîtriserait plus totalement. Faut-il choisir entre le chant des sirènes informatiques et le spectre de l'apprenti sorcier? Faut-il perdre son âme de traducteur pour gagner sa vie en traduisant? La déshumanisation de la fonction du traducteur est bel et bien redoutée: tel est le constat qu'a pu faire chaque participant, quelles que soient par ailleurs ses opinions. Les optimistes verront, en la matière, la nécessité d'une vaste action pédagogique en direction des formateurs de futurs traducteurs.

Dans les locaux d'une école chargée de former des interprètes, devant un auditoire composé d'un grand nombre d'enseignants, et dans le cadre de journées scientifiques d'une institution qui revendique à bon droit son titre d'université, la question de la formation des traducteurs de l'an 2001 ne pouvait pas être éludée. Plusieurs communications y ont été consacrées. Si certaines étaient nettement yolontaristes alors que d'autres s'en tenaient à une grande prudence programmatique, toutes ont fait ressortir la nécessité d'une formation solide et variée, meilleur gage de la valorisation de la profession. Inclure dans le cursus une réflexion plus approfondie sur la nature et le fonctionnement des langues de spécialité et de leur terminologie, connaître et savoir évaluer de manière critique les outils (machines, logiciels, documentation) et le milieu professionnel (marchés, entreprises, etc.), telles ont été les exigences jugées le plus souvent indispensables à une formation qui doit contribuer à la création d'une image du futur traducteur comme homme de communication multilingue.

Plusieurs communications d'orateurs venus des pays du sud ont aidé à la mise en perspective de ces problèmes de formation, notamment lorsqu'elle doit inclure toutes les spécificités locales. On a ainsi mesuré à quel point le rôle que la recherche peut y jouer est capital, notamment parce que celle-ci redonne à tout le processus une dimension essentiellement humaine et éloigne les risques d'aliénation mécaniste. L'organisation de programmes de recherche partagée et de stages de formation ou de recyclage a été évoquée à plusieurs reprises au cours de ces trois journées: elle constitue d'ailleurs l'un des objectifs prioritaires du Réseau lexicologie terminologie traduction.

Ces journées scientifiques avaient évidemment pour objet l'échange d'information sur l'état de la question et les perspectives de développement ainsi que le resserrement des liens entre chercheurs, concepteurs et praticiens. Elles devaient aussi conduire à des propositions d'actions et à des recommandations destinées au Comité scientifique du réseau. C'est à ce titre que trois tables rondes ont été organisées : sur les problèmes et les besoins de l'Afrique, sur les problèmes et les besoins du monde arabe et sur la problématique formationrecherche. Les trois rapporteurs ont fait des conclusions de leur groupe lors de la séance plénière de clôture.

Le colloque de Mons a été studieux mais, grâce à la diligence de son comité organisateur, il a été aussi fort agréable, notamment grâce à des réceptions diverses offertes par la ville de Mons, le gouvernement provincial et l'AUPELF-UREF. Tout ceci a assuré une relation harmonieuse entre les participants et leur environnement de travail et 
de détente. Il reste à souhaiter que, à l'instar des organisateurs de ces journées, le traducteur de l'an 2001 sache aménager et gérer son environnement de travail, afin de construire un écosystème traductionnel dont il restera, quoi qu'on en dise, l'élément central décisif.

PHILIPPE THOIRON Université Lumière, Lyon 2, Lyon France 